\title{
Moon illusions redescribed
}

\author{
DON McCREADY \\ University of Wisconsin-Whitewater, Whitewater, Wisconsin
}

\begin{abstract}
The common report that the horizon moon looks "larger and closer" than the zenith moon means that the perceived visual angle ( $V^{\prime}$ rad) for its diameter is greater, the perceived distance $\left(D^{\prime} \mathrm{m}\right)$ to it is less, and the perceived linear size $\left(S^{\prime} \mathrm{m}\right)$ for its diameter is either greater or the same (size constancy), all in accord with the rule $S^{\prime} / D^{\prime}=V^{\prime}$ rad. These majority moon illusions remain unexplained because published descriptions use only one "perceived size" variable, rather than both $V^{\prime}$ and $S^{\prime}$; and some create paradoxes by using the standard rule, $S^{\prime} / D^{\prime}=V \mathrm{rad}$, which omits $V^{\prime}$. Complete paradox-free redescriptions are offered, and the oculomotor explanation is outlined.
\end{abstract}

The full moon at the horizon often appears about 1.5 times wider than it does at its zenith, and a ratio as high as 2.0 is not uncommon (Enright, 1975; Holway \& Boring, 1940a, 1940b; Rock \& Kaufman, 1962a; Taylor \& Boring, 1942). Of course, the visual angle, $V=0.52^{\circ}$, subtended at the eye by the moon's azimuth diameter, remains virtually constant in accord with the rule

$$
V=S / D=0.009 \mathrm{rad} \text {. }
$$

$S$ is the moon's linear size (its diameter of $3,475 \mathrm{~km}$ ), and $D$ is its essentially constant distance from the eye (about $384,400 \mathrm{~km}$ ). For simple examples, $V$ (the optical direction difference) henceforth is rounded off to $0.01 \mathrm{rad}$.

To most observers, the larger looking horizon moon also looks closer than the smaller looking zenith moon. New attempts to explain this "larger and closer" illusion continue to appear, with each writer noting how previous explanations (theories) fail (Baird, 1982; Baird \& Wagner, 1982; Enright, 1975; Gilinsky, 1980; Hershenson, 1982; Loftus, 1985; Reed, 1984, 1985; Restle, 1970; Smith, Smith, Geist, \& Zimmermann, 1978). The present article $^{1}$ extends the argument (McCready, 1965, 1983, 1985) that published explanations fall short of the mark primarily because, in addition to the perceived distance variable, $D^{\prime} \mathrm{m}$, they use only one "perceived size" variable instead of both the perceived linear size value, $S^{\prime} \mathrm{m}$, and the perceived visual angle value, $V^{\prime}$ deg (the perceived optical direction difference). ${ }^{2}$ In other words, each published explanation addresses an incompletely described experience for the moon.

It seems clear that most observers' verbal report "looks larger and closer" refers first to the visual angle comparison $\left(V_{h}^{\prime}>V_{z}^{\prime}\right.$, with $\left.D_{h}^{\prime}<D_{z}^{\prime}\right)$, and also refers, secondarily, to the linear size comparison $\left(S_{h}^{\prime}>S_{z}^{\prime}\right.$, with

\footnotetext{
I thank 12 referees for their comments on my unpublished "moon illusion" manuscripts submitted since 1965. I am grateful to Richard Kelley, 1-Ning Huang, and Sally McCready for their continuing encouragement. I thank Lisa Horton for preparing the figures. Requests for reprints should be addressed to the Department of Psychology, University of Wisconsin-Whitewater, Whitewater, WI 53190.
}

$D_{h}^{\prime}<D_{z}^{\prime}$ ). Some observers may experience size constancy $\left(\mathrm{S}_{h}^{\prime}=\mathrm{S}_{z}^{\prime}\right)$, however, so "looks larger and closer" refers only to their visual angle and distance comparison and their experience of a constant linear diameter (and volume) remains unmentioned.

Although some investigators have used the variable $V^{\prime}$ deg (Baird, 1982; Enright, 1975; Restle, 1970; Rock \& Kaufman, 1962a, 1962b), most have agreed with the predominant theoretical assumption that $V$ is not perceived (Gilinsky, 1980; Hershenson, 1982; Reed, 1984).

\section{Size-Distance "Paradox"}

Moreover, many descriptions have been based upon the "size-distance invariance hypothesis" (SDIH), written as $S^{\prime} / D^{\prime}=k V$ rad, in which $k$ is an observer constant, nominally 1.0 (Gogel, 1977). Because the SDIH omits $V^{\prime}$, and because $k V$ rad is constant for the moon, the popular report "looks larger and closer" does not fit the SDIH and seemed to create a "size-distance paradox" (Epstein, Park, \& Casey, 1961). Recent explanations attempt to avoid that paradox but, by not using all three response variables, they often inadvertently recreate it.

New explanations will not be reviewed in detail here because the main purpose is simply to fully describe moon illusions using $V^{\prime}, S^{\prime}$, and $D^{\prime}$. Therefore, it is necessary to use an unconventional theory of spatial perception. I use the $s=d v$ theory (McCready, 1965, 1983, 1985).

\section{THE $s=d v$ THEORY APPROACH}

Figure 1 is a side view with the observer's eye at point $O^{\prime}$. The uppermost sphere illustrates the basic hypothesis stated by Equation 1:

$$
S^{\prime} / D^{\prime}=V^{\prime} \mathrm{rad}
$$

\section{Response Variables}

The perceived linear size, $S^{\prime} \mathrm{m}$, for a sphere is one's report about one's linear size experience $(s)$ for its diameter. For small-looking spheres, $S^{\prime}$ can be a haptic report, such as the distance one holds one's hands apart. But $S^{\prime}$ for the huge-looking moon must be a verbal esti- 


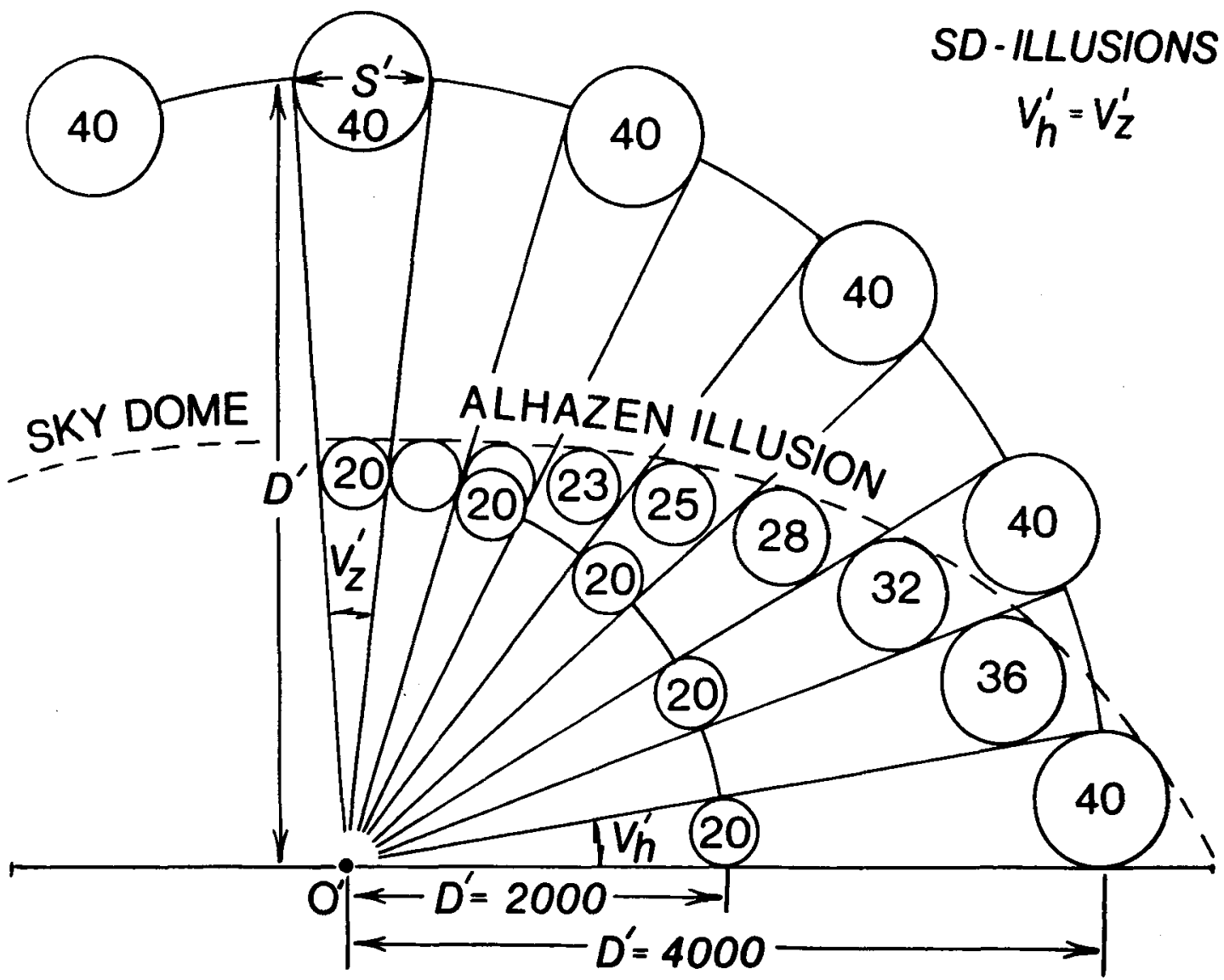

Figure 1. Perceived moons are shown in hypothetical examples for an observer who, at $O^{\prime}$, looks eastward (to the right) at the horizon moon and later looks upward at the zenith moon. The perceived visual angle $\left(V^{\prime}\right)$ remains 0.01 rad, so, in an $S D$ illusion, the perceived distance $\left(D^{\prime} \mathrm{m}\right)$ for every moon is 100 times the perceived linear size value $\left(S^{\prime} \mathrm{m}\right)$ for its diameter, as indicated with arbitrary units. The "Alhazen illusion" refers to the classic description in which, with $V_{h}^{\prime}$ $=V^{\prime}$ deg, the horizon moon looks both linearly larger and farther away than the zenith moon. However, relatively few observers suffer that "textbook" illusion.

mate. It undoubtedly is always considerably less than $3,475 \mathrm{~km}$, so an $S$ illusion always exists.

The perceived distance, $D^{\prime} \mathrm{m}$, is one's report about one's distance experience $(d)$. A haptic report about the hugelooking distance to the moon is ruled out, so $D^{\prime}$ must be a verbal estimate. It invariably is much less than $384,400 \mathrm{~km}$ (Gilinsky, 1980), so a $D$ illusion always exists.

The perceived visual angle value, $V^{\prime}$ deg, is one's report about one's visual angle experience (the visual direction difference, $v$ ). For the moon's azimuth diameter, this tiny subtense is only about $1 / 700$ th of a complete subjective directional rotation (nominally $360^{\circ}$ of compass directions) around oneself. Although $V^{\prime}$ deg could be a verbal estimate of $v$, a haptic estimate furnished by pointing responses may be preferred (Ono, 1970). For example, $V^{\prime}$ might be the initial head-rotation angle measured when one quickly aims one's nose from one edge of the moon to the opposite edge. (An accurate response moves the tip of the nose laterally only about $2 \mathrm{~mm}$.) Or, $V^{\prime}$ might be the ballistic eye-saccade angle obtained when one quickly looks from one edge to the other. (A veridical rotation moves the cornea laterally only about $0.15 \mathrm{~mm}$.)
For most observers, $v$ for the moon's diameter evidently decreases as the moon ascends (Baird, 1982; Enright, 1975; McCready, 1965, 1983, 1985; Restle, 1970; Rock \& Kaufman, 1962a, 1962b). Therefore, during all or most of the moon's trip, $V^{\prime}$ does not equal $V$ deg: $A V$ illusion usually exists. Unfortunately, no $V^{\prime}$ values have been published for the moon. Of course, it will be difficult to measure small variations in response angles that already are less than $1^{\circ}$. In research, the moon has been compared with other targets.

\section{Relative Equation}

From Equation 1, it follows that horizon to zenith moon comparisons are described by Equation 2:

$$
\left(S_{h}^{\prime} / S_{z}^{\prime}\right)\left(D_{z}^{\prime} / D_{h}^{\prime}\right)=V_{h}^{\prime} / V_{z}^{\prime} \text {. }
$$

The verbal report "looks larger" becomes unambiguous when a comparison disk (or disk image) at a fixed place is adjusted to look the "same size" as the moon seen first at the horizon and then at the zenith. On each trial, observers undoubtedly try to match their $v$ experiences for the moon and the disk (Enright, 1975; Rock 
\& Kaufman, 1962a, 1962b). The visual angle of the disk that looks the same as the moon is not a $V^{\prime}$ value either for the moon or for the disk. But the ratio of the disk's $V$ values which match the horizon and zenith moons properly can be recorded in Equation 2 as $V_{h}^{\prime} / V_{z}^{\prime}$. Now, if the horizon and zenith moons look equidistant $\left(D_{h}^{\prime}=D_{z}^{\prime}\right)$, Equation 2 becomes $S_{h}^{\prime} / S_{z}^{\prime}=V_{h}^{\prime} / V_{x}^{\prime}$. Therefore, if the ratio of the disk's $V$ values were mistakenly entered as $S_{h}^{\prime} / S_{z}^{\prime}$, as well as $V_{h}^{\prime} / V_{2}^{\prime}$, then no numerical error would appear, and the logical error of that entry might be overlooked. However, if $D_{h}^{\prime}$ and $D_{z}^{\prime}$ are unequal, which seems to be the usual case, then entry of the disks' ratio as $S_{h}^{\prime} / S_{\imath}^{\prime}$ would unbalance Equation 2, and the observer's responses would seem "paradoxical" if the entry error went unrecognized.

A complete description must specify all three ratios in Equation 2, but jointly obtained values for the $S^{\prime}$ and $D^{\prime}$ ratios have not been published. ${ }^{3}$

Before illusions with misperceived visual angles are considered, it is more convenient to describe potential " $S D$-illusions" in which $V_{h}^{\prime}=V_{2}^{\prime}$.

\section{RELATIVE SD-ILLUSIONS}

For every perceived sphere in Figure $1, V^{\prime}=0.01 \mathrm{rad}$ for the observer at $O^{\prime}$, so, for each one, $D^{\prime}=100 S^{\prime} \mathrm{m}$. Two "horizon" spheres lie east of $O^{\prime}$ (to the right). To simplify descriptions, Equation 2 is split into Equations $3 A$ and $3 B$,

$$
\begin{gathered}
V_{h}^{\prime} / V_{z}^{\prime}=1.0 \\
S_{h}^{\prime} / S_{z}^{\prime}=D_{h}^{\prime} / D_{z}^{\prime} .
\end{gathered}
$$

A true relative outcome is indicated by spheres on the arc at $D^{\prime}=2,000$ units (an equidistance outcome), so $S^{\prime}$ stays 20 units (an equisize outcome, or size constancy). Another is shown by spheres with $S^{\prime}=40$ units and $D^{\prime}$ $=4,000$ units.

\section{Relative $S D$-illusion}

Let a (trained) observer at $O^{\prime}$ say the horizon moon looks the same angular width, twice wider linearly, and twice as far away as the zenith moon. Spheres along the flattened arc illustrate an apparent path from horizon to zenith in which $S^{\prime}$ shrinks from 40 units to 20 units (an eightfold decrease in apparent volume) as $D^{\prime}$ decreases from 4,000 units to 2,000 units, while, of course, $V^{\prime}$ remains $0.01 \mathrm{rad}$.

An analogy is provided for the reader by these two equal circles, 00 , when, as a pictorial illusion, the left one appears to be a pictured ping-pong ball $\left(S^{\prime}=37 \mathrm{~mm}\right)$ and the right one appears to be a pictured baseball $\left(S^{\prime}=\right.$ $74 \mathrm{~mm}$ ), which, since they look the same angular width, looks twice as far away as the ping-pong ball.

Naive observers typically give just one of the two possible "size" and distance reports harbored in a full report comparing the horizon moon to the zenith moon. For the relative $S D$-illusion example in Figure 1 , the $S$-and- $D$ report is "looks twice wider and twice as far away," and the $V$-and- $D$ report is "looks the same width and twice as far away."

Relative $S D$-illusion descriptions include the incomplete classic description discussed below.

\section{Standard Approach}

In comparative form, the SDIH equation $\left(S^{\prime} / D^{\prime}=\right.$ $k V \mathrm{rad})$ is $S_{h}^{\prime} / D_{k}^{\prime}=S_{z}^{\prime} / D_{z}^{\prime}$, which expresses "Emmert's law" (King \& Gruber, 1962). Rearranged, it becomes Equation 4.

$$
S_{k}^{\prime} / S_{z}^{\prime}=D_{k}^{\prime} / D_{z}^{\prime} \quad \text { (The SDIH) }
$$

Because Equation 4 is the same as Equation 3B, SDIH descriptions must treat all moon illusions as relative $S D$ illusions. Indeed, if the primes on the $V^{\prime}$ symbols in Figure 1 are erased, it then resembles the classic "textbook" diagram, which overlooks Equation 3A and uses the equal stimulus values $\left(V_{h}=V_{z}\right)$ instead (Kaufman \& Rock, 1962; King \& Gruber, 1962; Restle, 1970).

Apparent distance explanation. $S D$ illusions like the one shown in Figure 1 may be called Alhazen moon illusions because, according to Ross and Ross (1976), Alhazen was the first to suggest that, in accord with Equation 4 , the rising moon appears to shrink because it appears to approach: He proposed that it appears to glide along an illusory sky surface shaped like a flat ceiling, but I take the liberty of generalizing his explanation to include, as shown, the familiar "sky dome" illusion."

Rock and Kaufman (1962a) improved that SDIH explanation by proposing that $D_{z}^{\prime}$ becomes less than $D_{h}^{\prime}$ because the zenith scene lacks the "great-distance" cues normally found in the horizon vista. They, and others since (Nelson \& Ladan, 1969), confirmed that the "largelooking" horizon moon will look smaller if those "greatdistance" cues are obscured or removed; and, conversely, that adding them near the "small-looking" zenith moon will make it look larger.

"Paradox." However, most observers say the larger looking horizon moon looks closer than the smaller looking zenith moon (Boring, 1962; Enright, 1975; Rock \& Kaufman, 1962a, 1962b). Moreover, this "paradoxical moon illusion" also occurs for the sun, for optical images, and for real targets placed in representative "horizon" and "zenith" stimulus configurations (Bilderback, Taylor, \& Thor, 1964; Conum, 1976; Gruber, King, \& Link, 1963; Iavecchia, Iavecchia, \& Roscoe, 1983; Leibowitz \& Hartman, 1959, 1960; Nelson \& Ladan, 1969; Orbach \& Solhkhah, 1968; Thor, Winters, \& Hoats, 1969). ${ }^{5}$

Clearly, majority moon illusions are not relative $S D$ illusions: They are relative VSD illusions.

\section{RELATIVE VSD ILLUSIONS}

To record "looks larger" as $V_{h}^{\prime}>V_{z}^{\prime}$ deg means that the illusion is as if the horizon moon's retinal-image diameter $\left(R_{h} \mathrm{~mm}\right)$ were larger than the zenith moon's $\left(R_{z} \mathrm{~mm}\right)$. Indeed, people familiar with optics usually are 


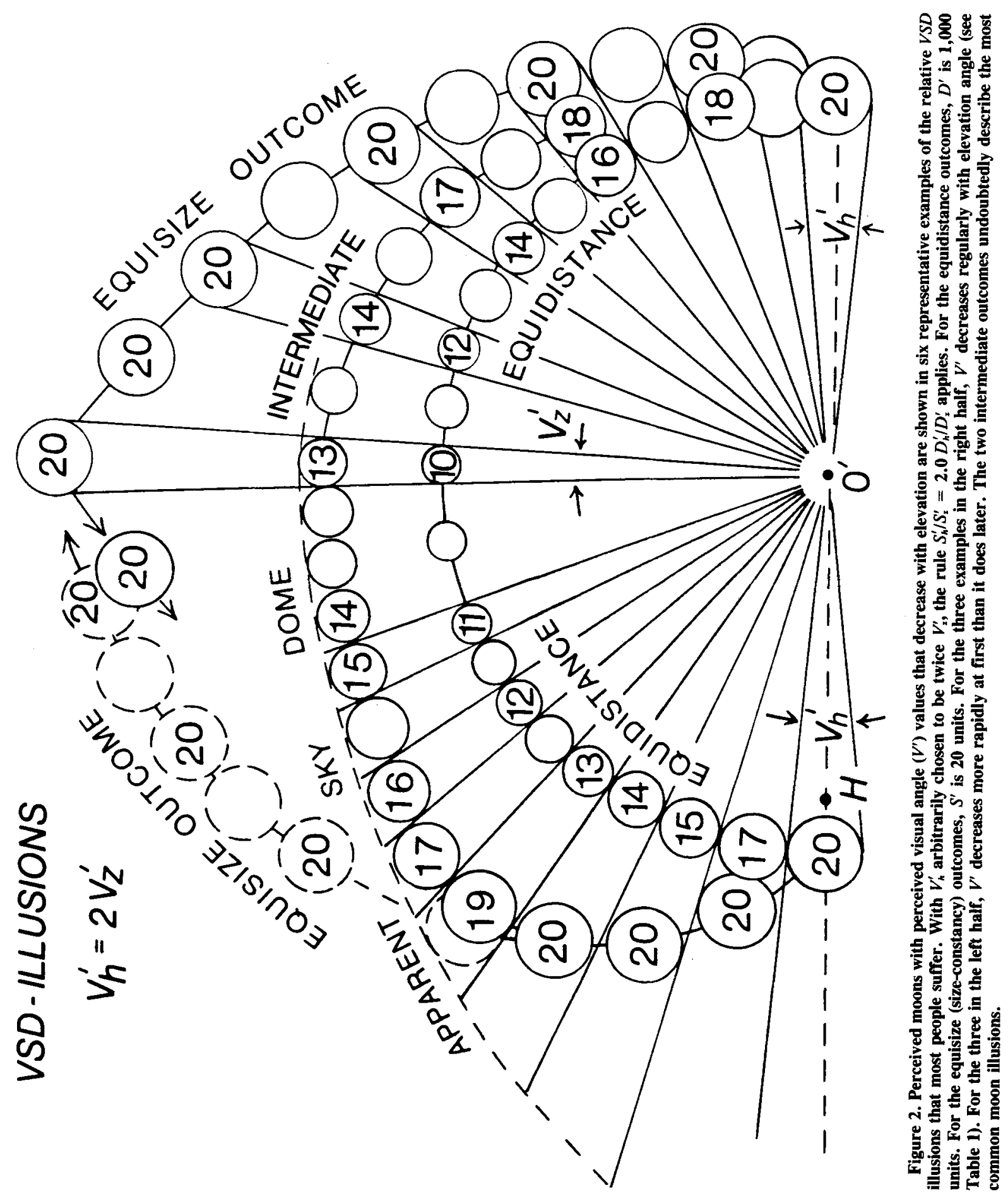


quite skeptical when they first learn that $R_{h}=R_{z}$ (about $0.15 \mathrm{~mm}$ ). Recalling their moon perceptions, they often insist that $R_{h}$ must exceed $R_{x}$, either because $V_{h}$ exceeds $V_{x}$ due to an atmospheric refraction or (if they accept that $V_{h}=V_{z}$ ) because of a change in the eye's imaging properties. Enright (1975) confirmed that, even with accommodation errors, $R_{h}$ and $R_{x}$ do not differ enough to account for the illusion.

To describe some relative $V S D$-illusion examples with "looks twice wider" recorded as $V_{h}^{\prime}=2.0 V_{z}^{\prime} \mathrm{deg}$, it helps to split Equation 2 into Equations $6 \mathrm{~A}$ and $6 \mathrm{~B}$.

$$
\begin{gathered}
V_{h}^{\prime} / V_{z}^{\prime}=2.0, \\
S_{h}^{\prime} / S_{z}^{\prime}=2.0 D_{h}^{\prime} / D_{z}^{\prime} .
\end{gathered}
$$

Six hypothetical examples appear in Figure 2. The horizon moon values arbitrarily are $V_{h}^{\prime}=0.02 \mathrm{rad}, S_{h}^{\prime}=$ 20 units, and $D_{h}^{\prime}=1,000$ units. For the zenith moon, $V_{z}^{\prime}$ $=0.01 \mathrm{rad}$, so it is drawn half the $V_{h}^{\prime}$ value. ${ }^{6}$

\section{Regular Decrease in $V^{r}$}

In the right half of Figure 2, the $V^{\prime}$ values decrease regularly with the increase in elevation (see Table 1, columns 1 and 2). The two most economical perceptual outcomes are the equidistance and equisize outcomes.

Equidistance outcome. Spheres on the arc at $D^{\prime}$ $=1,000$ units illustrate an equidistance outcome that requires that the reduction in $S^{\prime}$ (from 20 units to 10 units) match the reduction in $V^{\prime}$ (see Table 1 , column 3 ). The observer at $O^{\prime}$ says the horizon moon looks twice wider angularly, twice wider linearly, and the same distance as the zenith moon.

From that full report can come a naive $V$-and- $D$ report, "looks larger and the same distance away," and a naive $S$-and- $D$ report, "looks larger and the same distance away," which sounds the same but is qualitatively different. An analogy is the pictorial illusion for these two circles, $\circ \mathrm{O}$, when the left one appears to be a pictured pingpong ball and the right one a pictured baseball that looks

Table 1

Arbitrary Values of $V^{\prime}, S^{\prime}$, and $D^{\prime}$ as a Function of the Moon's Elevation Angle for Examples Illustrated in Figure 2

\begin{tabular}{ccccc}
\hline \multicolumn{3}{c}{ Values for the Right Half of Figure $2^{*}$} & \\
\cline { 1 - 3 } $\begin{array}{c}\text { Elevation } \\
\text { Angle }\end{array}$ & $V^{\prime}$ rad & $S^{\prime}$ if $D^{\prime}=$ & $D^{\prime}$ if $S^{\prime}=$ & \\
\hline $0^{\circ} \ddagger$ & .000 units & 20 units & For Left Half $\dagger$ \\
$9^{\circ}$ & .019 & 20 & 1,000 & $0^{\circ}$ \\
$18^{\circ}$ & .018 & 19 & 1,053 & - \\
$27^{\circ}$ & .017 & 17 & 1,111 & - \\
$36^{\circ}$ & .016 & 16 & 1,176 & $9^{\circ}$ \\
$45^{\circ}$ & .015 & 15 & 1,250 & - \\
$54^{\circ}$ & .014 & 14 & 1,333 & $18^{\circ}$ \\
$63^{\circ}$ & .013 & 13 & 1,429 & $27^{\circ}$ \\
$72^{\circ}$ & .012 & 12 & 1,538 & $36^{\circ}$ \\
$81^{\circ}$ & .011 & 11 & 1,667 & $54^{\circ}$ \\
$90^{\circ} \S$ & .010 & 10 & 1,818 & $72^{\circ}$ \\
\hline
\end{tabular}

*With a regular change in $v$. †Approximate elevation angles for irregular change in $V . \ddagger$ Horizon. \$Zenith. twice wider angularly, twice wider linearly (hence eight times the volume), and the same distance away as the pingpong ball.

Equisize outcome. An equisize outcome (size constancy) is illustrated by spheres which, with $S^{\prime}=20$ units, must follow a pointed-horseshoe arch because the $D^{\prime}$ values are inversely proportional to the $V^{\prime}$ values (see Table 1 , column 4). The observer says the horizon moon looks twice wider angularly, the same linear diameter (and same volume), and half as far away as the zenith moon.

The $V$-and- $D$ report is "looks larger and closer," and the $S$-and- $D$ report is "looks the same size and closer." An analogy is the pictorial size-constancy illusion for these two circles, $\odot$, when both appear to be pictured baseballs: The right baseball looks the same linear width, twice wider angularly, and half as far away as the left one.

Figure 2 reveals that the rising moon, during the first part of its trip in the size-constancy outcome, would appear to ascend vertically, as if it were a rising balloon, except for a slight bulge away from a truly vertical path.

Intermediate outcomes. At each elevation angle, spheres having the given $V^{\prime}$ value at that elevation can be inserted between the spheres on the equidistance and equisize paths to illustrate intermediate outcomes. The illustrated intermediate path for the rising moon leads to the perceived zenith moon with $S^{\prime}=13$ units and $D^{\prime}=$ 1,300 units. Compared with all possible intermediate zenith moons, the horizon moon looks angularly twice wider, closer, and linearly wider, but not as much as twice wider. Indeed, as a general rule for all intermediate outcomes with $V_{h}^{\prime}>V_{x}^{\prime}$, all the $V$-and- $D$ reports and $S$-and$D$ reports are "looks larger and closer"': Both incomplete reports now sound like the majority reports.

A conclusion. Majority moon illusions undoubtedly are intermediate relative VSD illusions. Indirect evidence is that most observers do not seem to realize that their simple report "looks larger and closer" is incomplete. The fact that they seem satisfied with mentioning only one "size" comparison suggests that the closer looking horizon moon looks larger than the zenith moon both angularly and linearly. Otherwise, if most observers suffered the size-constancy outcome $\left(V_{h}^{\prime}>V_{z}^{\prime}\right.$ and $\left.S_{h}^{\prime}=S_{z}^{\prime}\right)$, then more of them would be expected to ask which "size" comparison they should report, the "unequal-looking" angular subtenses or the "equal-looking" linear sizes (see Joynson, 1949).

Better evidence that majority moon illusions are intermediate relative VSD illusions is that some audience members objected to my early Figure 2 diagrams, which showed only the equidistance and equisize paths; they insisted that the horizon moon usually appeared both closer than the zenith moon and to have a larger volume, thus $S_{h}^{\prime}>S_{z}^{\prime}$. Since then, informal polls at the end of my lectures indicate that, as Loftus (1985) also notes, most people see it that way. That outcome also means, of course, that $V_{h}^{\prime}>V_{z}^{\prime}$.

Other outcomes. Many other general outcomes that conform to Equations $6 \mathrm{~A}$ and $6 \mathrm{~B}$ are not included in 
Figure 2. For example, if the horizon moon looked farther than the zenith moon $\left(D_{h}^{\prime}>D_{z}^{\prime}\right)$, then the ratio $S_{h}^{\prime} / S_{z}^{\prime}$ would be greater than the ratio $V_{h}^{\prime} / V_{z}^{\prime}$. Both the $V$-and- $D$ and $S$-and- $D$ reports would be "looks larger and farther," an infrequent report.

\section{Nonuniform Decrease in $V$}

To some observers (perhaps most), the rising moon's "apparent size" decreases more rapidly just after it leaves the horizon than it does later (Enright, 1975; Holway \& Boring, 1940a, 1940b; Rock \& Kaufman, 1962a). Three more examples with $V_{h}^{\prime}=2.0 V_{2}^{\prime}$, but with an arbitrarily chosen irregular decrease in $V^{\prime}$ deg, appear in the left half of Figure 2 (left is now eastward). Approximate elevations for these spheres are listed in the last column of Table 1.

On the equidistance arc, the $S^{\prime}$ values indicate the irregular change in $V^{\prime}$ for all three examples.

The equisize outcome gives an arch broader than the one in the right half of Figure 2: The apparent retreat of the moon to a greater distance just after it rises is more dramatic. This illusory retreat, and its complement (illustrated by an apparently rapid approach of the setting sun), was mentioned by Hershenson (1982, p. 438, Footnote 7).

Intermediate example. The final example is an intermediate outcome that also illustrates three suggestions Gilinsky (1980) offered as part of her rejection of the horizon part of the classic "sky dome' description. First, the horizon sky looks much farther away than the farthest looking "horizon" terrestrial objects indicated by point $H$ in Figure 2. Second, the horizon moon appears to be not "on" the sky surface, but only slightly beyond the objects at point $\mathrm{H}$. Last, the zenith sky and zenith moon both appear closer than the horizon sky, but slightly farther than the horizon moon.

In my example, the rising moon appears to follow the size-constancy path $\left(S^{\prime}=20\right)$ until it almost reaches the "locus at which sky and moon meet in visual space", (Gilinsky, 1980, p. 208). Then it appears to move as if along a flattened arc; $S^{\prime}$ decreases because both $V^{\prime}$ and $D^{\prime}$ are decreasing. At the zenith, it looks angularly half as wide as the horizon moon, linearly smaller $\left(S_{z}^{\prime}=\right.$ 13 units), and slightly farther away ( $D_{z}^{\prime}=1,300$ units).

That description does not agree with Gilinsky's, which, because it uses the SDIH and only one "perceived size" variable, requires that the farther looking zenith moon look larger than the closer looking horizon moon. The "paradox" is not avoided.

Reed (1984) offered, in his Figure 1, an "apparent path" that resembles an intermediate path in my Figure 2. But his path shows $D^{\prime}$ values furnished by equations that do not include $V^{\prime}$ or $S^{\prime}$. Thus, with $V$ constant, the $S^{\prime}$ values for apparent moons along that path would increase with both $D^{\prime}$ and the elevation angle. The paradox remains.

Moon illusion descriptions by Hershenson (1982) and Loftus (1985) also do not use both $V^{\prime}$ and $S^{\prime}$ along with $D^{\prime}$.
Countless apparent path shapes fit within the intermediate $V S D$-illusion description. For example, after the moon appears to reach a sufficient linear altitude, its apparent path through most of the zenith sky could agree with Alhazen's "flat ceiling" and still have $D_{z}^{\prime}>D_{h}^{\prime}$, as would be indicated in Figure 2 by a horizontal line at the height of the circle with $S^{\prime}=16$ units on an intermediate path.

\section{Illustrations Mislead}

Diagrams that, like Figure 2, show unequal $V^{\prime}$ values for the horizon and zenith moons, have been presented only outside journals and books (see Footnote 1). On the other hand, the standard diagram (Kaufman \& Rock, 1962), which Figure 1 resembles, has been republished often, even though it fails to describe most readers' moon illusions. Its wide acceptance indicates that it has been widely misread.

Artifacts. When viewing the "textbook" figure, a student may suffer pictorial artifacts if he or she forgets it is a side view in which the reader's $v$ and $s$ experiences for the circles often disagree with the sphere experiences being portrayed for the observer at $O^{\prime}$. For example, for Figure 1, the reader's "angularly larger" experience for the large "horizon" circle (40 units), relative to the small "zenith" circle (20 units), mimics the majority moon illusion experience: Therefore, because this $v$ experience "rings true" for most students, they might mistakenly assume that Figure 1 is illustrating a similar experience for the observer at $O^{\prime}$. However $V_{h}^{\prime}$ and $V_{s}^{\prime}$ deg are deliberately drawn equal in order to illustrate that, to the observer at $O^{\prime}$, those spheres look angularly equal.

In Figure 2, the equal circles $\left(S^{\prime}=20\right)$ on the sizeconstancy paths all have the same $v$ value for the reader but illustrate spheres which have changing $v$ values for the observer at $O^{\prime}$. Thus, a student might be misled into thinking that size constancy refers to $v$.

It is still necessary to explain the illusions.

\section{EXPLAINING MOON ILLUSIONS}

The major problem is to explain first why $V_{h}^{\prime}$ exceeds $V_{x}^{\prime}$ in majority moon illusions. It then only remains to use a logical model of visual processing (see Rock, 1977, or McCready, 1985) to explain why, with $V_{k}^{\prime} / V_{z}^{\prime}>1.0$ in Equation 2, the ratios of $S^{\prime}$ and $D^{\prime}$ values become an equidistance outcome, a size-constancy outcome, or, more often, an intermediate outcome.

Because published explanations use only one "size" response variable, explanations for the $V$ illusion have been confused with explanations for the $S$ illusion that usually accompanies it. ${ }^{7}$ Consider just the $V$ illusion.

\section{$V$-Contrast Description}

One explanation that treats "perceived size" as $V$ ' deg, and avoids using the SDIH (Equation 4), is Restle's (1970) adaptation-level theory model. It overlooks $S^{\prime}$ values, but describes a visual-angle contrast ( $\boldsymbol{V}$-contrast) illusion in which the moon's visual angle appears to decrease when 
there is an increase in the visual angles subtended between the moon's rim and adjacent context contours. In present terms, Restle's relative equation is,

$$
V_{h}^{\prime} / V_{x}^{\prime}=A_{V_{z}} / A_{V h},
$$

in which the "zenith" adaptation level value, $A_{V_{z}}$ deg, is a weighted geometric mean of context $V$ values near the zenith moon, and $A_{V h}$ deg is the "horizon" adaptation level for $V$-context values near the horizon moon. Because the illusion is that $V_{h}^{\prime}$ exceeds $V_{z}^{\prime}$, "contrast" theorists select context contours that will give $A_{V x}$ greater than $A_{V h}$ deg: the huge angular expanse of "empty" zenith sky is pitted against the fine texture of tiny $V$ values among terrestrial contours near the horizon.

Baird (1982) noted that his "sky model" is essentially the same as Restle's model. Rizzo (1963) and Smith et al. (1978) also advocated this "relative size" description. (In standard theories, the equivocal term "relative size" often refers to $V^{\prime}$ deg which, according to those same theories, does not exist.)

$V$-contrast approaches usefully describe the change in $V^{\prime}$ for the moon, but do not explain why an increase in $V$-context values would decrease $V^{\prime}$, rather than leaving it the same or increasing it (as $V$ assimilation). Two general explanations seem most plausible.

\section{Contour (Neural) Interactions}

One type of explanation redescribes $V$-contrast effects as contour interaction illusions (see Over, 1968) which are being explained neurologically, either in terms of interactions among the cortical neural activities generated by the retinal images of the target and context contours or in terms of adaptation of spatial frequency detectors (for a review, see Coren \& Girgus, 1978).

However, many investigators have pointed out that contour (neural) interactions cannot account for all of the moon illusion, and an additional explanation must give distance "cues" a more direct role. For example, the "larger" $V_{h}^{\prime}$ value for the horizon moon shrinks when the visual scene is inverted (Enright, 1975; Rock \& Kaufman, 1962a), but the inversion, if properly done, does not alter the contour relationships near the moon; $A_{V h}$ is unchanged.

Distance cue control. $V$-context patterns are, of course, "texture gradient" and linear perspective patterns which illustrate the rule $D^{\prime}=S^{\prime} / V^{\prime}$. A decrease in $V^{\prime}$ rad between contours yields an increase in $D^{\prime} \mathrm{m}$ if, as size constancy, $S^{\prime} \mathrm{m}$ is scaled about the same for those contour separations. Moon illusions and many other illusions demonstrate that a substantial decrease in those $V$-context values and the presence of monocular cues to a large increase in a target's distance induce a slight increase in $V^{\prime}$ for the target's constant $V$ value.

Moreover, as Rock, Shallo, and Schwartz (1978) showed, the more an observer recognizes, interprets, and accepts that a viewed pattern indicates large depth values (large distance differences), the more $V^{\prime}$ increases for a target of constant $V$ located at a nominally "far" place in the visual world. A complementary illusion is that in- verted viewing of the landscape typically makes all horizon objects look smaller and farther away (Washburn, 1894): The inversion minifies all $v$ values, including those for buildings, trees, hills, clouds, and the moon. Restoring the upright scene, with its familiar arrangements of contextual "cues" to great depths, then magnifies $v$ values back to their former values.

The basic problem thus is to explain why distance-cue variables control the ratio $V^{\prime} / V$. An oculomotor explanation (McCready, 1983) is outlined below.

\section{Oculomotor Explanation}

The second type of explanation for $V$-contrast illusions turns to an examination of the illusions of accommodationconvergence micropsia (AC micropsia), which also demonstrate control of $V^{\prime} / V$ by distance cue variables. A satisfactory explanation of AC micropsia exists (Komoda \& Ono, 1974; McCready, 1965, 1983, 1985; Ono, 1970; Ono et al., 1974). [The explanation for AC micropsia outlined below is elaborated in a paper (I have in preparation) that needs to be published before a paper that applies it to moon illusions.]

AC Minification. An increase in oculomotor efference (AC efference), aimed toward shifting accommodation and convergence to a closer point, shrinks $V^{\prime}$ for a constant $V$, to illustrate AC minification, which is the initial $V$ illusion in AC micropsia. AC minification seems to be a perceptual adjustment that corrects for the difference between the rotation angles that will accurately orient the head and the eye from one nearby target point to another, $V$ deg away. This discrepancy occurs because headrotation centers lie about 10 or $15 \mathrm{~cm}$ posterior to an eyerotation center. Thus, for two points close to the eye and subtending $V$ deg, an accurate eye-saccade angle from one to the other must equal $V \mathrm{deg}$, but a head rotation that will accurately orient the head (thus both eyes and both ears) squarely from one point to the other must be less than $V$ deg. Yet, both of those $V^{\prime}$ values operationally define the same visual-direction-difference value $(v)$ : So, if they are equal for a given $v$, one of them must be inaccurate.

Evidently, $v$ is kept a more accurate predictor of head rotations than of eye rotations, and $\mathrm{AC}$ efference mediates (controls) the required shift of $v$ away from being equal to $V$ deg. If so, Equation 7 , below, specifies the amount by which $V^{\prime}$ should become less than $V$ for an approaching frontal extent if the eyes accurately focus and converge upon it.

$$
V^{\prime} / V=D_{C}^{\prime} /\left(D_{C}^{\prime}+T_{K}\right)
$$

The variable $D_{c}^{\prime} \mathrm{cm}$ is the distance to which the eyes have been accommodated and converged. For present purposes, $D_{c}^{\prime}$ also can express (reciprocally) the magnitude of $\mathrm{AC}$ efference directed toward those AC responses.

The value $T_{k} \mathrm{~cm}$ is an observer constant that theoretically should equal the distance between a head-rotation center and an eye-rotation center. Indeed, with $T_{K}$ values 
ranging from 5 to $15 \mathrm{~cm}$, Equation 7 fits much published data that reveal AC minification (see McCready, 1965). (Needed revisions of Equation 7, and its applications to recent data, will be discussed elsewhere.)

\section{AC Minification in Moon Illusions}

Enright (1975) listed variables which could create ACefference differences between the horizon and zenith viewing conditions, such that $D_{C}^{\prime}$ would be greater for the horizon moon than for the zenith moon. In that case, AC minification would be expected to render $V_{h}^{\prime}$ greater than $V_{z}^{\prime}$. Recently, Iavecchia et al. (1983) found that the "apparent size" (clearly, $V^{\prime} \mathrm{deg}$ ) for a simulated rising "moon" varied directly with the measured distance $\left(D_{C}^{\prime}\right)$ to which the eye was accommodated. (To a first approximation, the comparative form of Equation 7, with $T_{K}$ about $12 \mathrm{~cm}$, fits some of their results. A fuller analysis will require modifying Equation 7 to take into account the "dark focus" values of $D_{c}^{\prime}$.)

Cue control. During normal viewing, the changing AC responses required for clear single binocular vision of nearby targets at different distances are accompanied by specific predictable changes in "distance cue" patterns. This routine correlation evidently maintains a relationship (say a conditioned reflex) such that distance cue changes in any display can evoke changes in AC efference (as conditioned responses) whether or not a change in $\mathrm{AC}$ responses is appropriate. So, even for two-dimensional patterns, such as photographs and many geometrical illusions, and for all targets at great distances (beyond the need for different AC responses), the "monocular cues" to a greater (lesser) distance for a given extent can inappropriately increase (decrease) $D_{c}^{\prime}$ for it and thereby unnecessarily increase (decrease) the ratio $V^{\prime} / V$.

Conditioned AC minification. A complication is that the ratio $V^{\prime} / V$ evidently may differ for two simultaneously viewed targets for which $\mathrm{AC}$ efference obviously must be the same at any moment. Therefore, it is necessary to propose, further, that, in the link between distance cues and the ratio $V^{\prime} / V$, the change in $\mathrm{AC}$ efference (thus in $D_{c}^{\prime}$ ) can be bypassed: Distance cues, including V-contrast patterns that might create contour (neural) interactions, may also directly evoke a conditioned AC minification.

In a later paper, I hope to elaborate the hypothesis that AC minification evoked by distance cues is the major source of the $V$ illusions that initiate majority moon illusions.

\section{REFERENCES}

BAIRD, J. C. (1982). The moon illusion: II. A reference theory. Journal of Experimental Psychology: General, 111, 304-315.

BaIRD, J. C., \& WaGNer, M. (1982). The moon illusion: I. How high is the sky? Journal of Experimental Psychology: General, 111, 296-303.

Bilderback, L. G., TAylor, R. E., \& ThOR, D. H. (1964). Distance perception in darkness. Science, 145, 294-295.

Boring, E. G. (1962). On the moon illusion [Letter to the editor]. Science, 137, 902-906.
Coren, S., \& GiRgus, J. S. (1978). Seeing is deceiving: The psychology of visual illusions. New York: Halsted.

CoRUM, M. C. (1976). On the moon illusion (Rand Paper P-5679). Santa Monica, CA: The Rand Corporation.

ENRIGHT, J. T. (1975). Moon illusion examined from a new point of view. Proceedings of the American Philosophical Society, 119, 87-107.

EPSTEIN, W., PARK, J., \& CASEY, A. (1961). The current status of the size-distance hypothesis. Psychological Bulletin, 58, 491-514.

Gilinskx, A. (1980). The paradoxical moon illusions. Perceptual \& Motor Skills, 50, 271-283.

GOGEL, W. C. (1977). The metric of visual space. In W. Epstein (Ed.), Stability and constancy in visual perception: Mechanisms and processes (pp. 129-181). New York: Wiley.

Gruber, H. E., King, W. L., \& Link, S. (1963), Moon illusions: An event in imaginary space. Science, 139, 750-752.

HERSHENSON, M. (1982). Moon illusion and spiral aftereffect: Illusions due to the loom-zoom system? Journal of Experimental Psychology: General, 111, 423-440.

Holway, A. H., \& Boring, E. G. (1940a). The moon illusion and the angle of regard. American Joumal of Psychology, 53, 109-116.

Holway, A. H., \& Boring, E. G. (1940b). The apparent size of the moon as a function of the angle of regard: Further studies. American Journal of Psychology, 53, 537-553.

Iavecchia, I. H., Iavecchia, H. P., \& Roscoe, S. N. (1983). The moon illusion revisited. Aviation, Space, \& Environmental Medicine, 54, 39-46.

Joynson, R. B. (1949). The problem of size and distance. Quarterly Journal of Experimental Psychology, 1, 119-135.

Kaufman, L., \& Rock, I. (1962). The moon illusion, I. Science, 136, 953-961.

King, W. L., \& Gruber, H. E. (1962). Moon illusion and Emmert's law. Science, 135, 1125-1126

KOMODA, M. K., \& ONO, H. (1974). Oculomotor adjustments and sizedistance perception. Perception \& Psychophysics, 15, 353-360.

Leibowitz, H., \& Hartman, T. (1959). Magnitude of the moon illusion as a function of the age of the observer. Science, 130, 569-570.

LeIBowitz, H., \& HaRTMAN, T. (1960). Reply to Cohen [Letter to the editor]. Science, 131, 694.

LoftUs, G. R. (1985). Size illusion, distance illusion, and terrestrial passage: Comment on Reed. Joumal of Experimental Psychology: General, 114, 119-121.

MCCREADY, D. (1965). Size-distance perception and accommodationconvergence micropsia: A critique. Vision Research, 5, 189-206.

MCCREADY, D. (1983). Moon illusions and other visual illusions redefined (Psychology Department Report). Whitewater: University of Wisconsin-Whitewater.

MCCREADY, D. (1985). On size, distance, and visual angle perception. Perception \& Psychophysics, 37, 323-334.

Nelson, T. M., \& LADAN, C. J. (1969). Size perceptions under several field conditions. American Journal of Optometry \& Archives of American Academy of Optometry, 46, 418-425.

ONO, H. (1970). Some thoughts on different perceptual tasks related to size and distance. In J. C. Baird (Ed.), Human space perception: Proceedings of the Dartmouth Conference. Psychonomic Monograph Supplement, 3(13, Whole No. 45), 143-151.

Ono, H., Muter, P., \& Mrtson, L. (1974). Size-distance paradox with accommodative micropsia. Perception \& Psychophysics, 15, 301-307.

OrBaCH, J., \& SolmKHaH, N. (1968). Size judgments of disks presented against the zenith sky. Perceptual \& Motor Skills, 26, 371-374.

OVER, R. (1968). Explanations of geometrical illusions. Psychological Bulletin, 70, 545-562.

REED, C. F. (1984). Terrestrial passage theory of the moon illusion. Journal of Experimental Psychology: General, 113, 489-500.

REED, C. F. (1985). More things in heaven and earth: A reply to Loftus. Journal of Experimental Psychology: General, 114, 122-124.

RESTLE, F. (1970). Moon illusion explained on the basis of relative size. Science, 167, 1092-1096.

Rizzo, P. (1963, July). Relativity and the moon illusion. The Eye-Piece, Amateur Astronomers Association, pp. 5-6.

Rock, I. (1977). In defense of unconscious inference. In W. Epstein 
(Ed.), Stability and constancy in visual perception: Mechanisms and processes (pp. 321-373). New York: Wiley.

Rock, 1., \& Kaufman, L. (1962a). The moon illusion, II. Science, 136, 1023-1031.

Rock, I., KAUFMAN, L. (1962b). On the moon illusion [Letter to the editor]. Science, 137, 906-911.

Rock, I., Shallo, J., \& Schwartz, F. (1978). Pictorial depth and related constancy effects as a function of recognition. Perception, 7 , 3-19.

Ross, H. E., \& Ross, G. M. (1976). Did Ptolemy understand the moon illusion? Perception, 4, 377-385.

Smith, O. W., Smith, P. C., Geist, C. C., \& Zimmermann, R. R. (1978). Apparent size contrasts of retinal images and size constancy as determinants of the moon illusion. Perceptual \& Motor Skills, 46, 803-808.

TAYLOR, D. W., \& BoRING, E. G. (1942). The moon illusion as a function of binocular regard. American Joumal of Psychology, 55, 189-201.

Thor, D. H., WinTers, J. J., \& HoATs, D. L. (1969). Vertical eye movement and space perception: A developmental study. Journal of Experimental Psychology, 82, 163-167.

WASHBURN, M. (1894). The perception of distance in the inverted landscape. Mind, n.s. 3, 438-440.

\section{NOTES}

1. The present article derives from a lecture, "The Moon Illusion Problem," presented to colloquia at the University of Chicago (1964), Marquette University (1968), Lawrence University (1970), and the University of Wisconsin-Whitewater $(1970,1981)$. It also evolves from manuscripts submitted in 1965, 1981, 1982a, 1982b, 1983, and 1984, but not published.

2. Some confusion may persist because, in psychology, "perceived" and "'apparent" are synonyms for the response measures, $S^{\prime} \mathrm{m}, D^{\prime} \mathrm{m}$, and $V$ ' deg, but in astronomy "apparent" refers to the stimulus value, $V$ deg: The moon's "apparent diameter," $V$, is $0.52^{\circ}$, and its linear diameter, $S$, is $3,475 \mathrm{~km}$. In present terms, a verbal report that the moon looks $100 \mathrm{~m}$ wide is recorded as the perceived (or apparent) linear size value $\left(S^{\prime}=100 \mathrm{~m}\right)$, and a report that its opposite edges look directionally different by $.5^{\circ}$ is recorded as the perceived (or apparent) visualangle value $\left(V^{\prime}=0.5^{\circ}\right)$. An astronomer, but not a psychologist, could call $V^{\prime}$ " "perceived apparent size" or even "apparent apparent size" without being redundant.

3. Many difficulties arise because most people do not clearly verbalize the difference between their linear size experience $(s)$ and direction difference experience $(v)$ for the moon's width. For example, consider a size-constancy outcome $\left(S_{h}=S_{z}\right)$ : Instead of using different adverbs to say the horizon moon "looks angularly larger but linearly the same size" as the zenith moon, naive observers are likely to use different verbs in a phrase such as "it looks larger, but I know it is the same size" (Joynson, 1949; Rock, 1977). Here "look" refers to $v$ and "know" refers to $s$, but the phrase could just as well refer entirely to the $v$ comparison ("The visual angle looks larger but I know it is constant"); and, to complicate matters, the same phrase also could refer entirely to the $s$ comparison in a different experience ("The linear diameter looks larger, but I know it is constant"'). A researcher obviously must know which experience the phrase describes.

Now consider that, in present terms, the appropriate report for the most common illusion is that the horizon moon "looks angularly larger and linearly larger" than the zenith moon. In this case, the naive use of different verbs would yield the ill-fitting statement, "it looks larger and I know it is larger," which an observer is unlikely to say. Indeed, naive observers should find the majority illusion impossible to verbalize without using different adverbs (or adjectives) to distinguish between their $v$ and $s$ experiences. Evidently, most observers, by default, are letting the incomplete ambiguous report "looks larger" do double duty. To provide unequivocal reports, they have to realize that they see not only a linear width, but also a direction difference value.

4. The difference between our frequent exposure to things passing overhead in flat trajectories, and our infrequent exposure to things passing over in arched trajectories, is important, however, to Reed's $(1984,1985)$ "terrestrial passage" theory of the moon illusion.

5 . Until reliable assessments of the relative proportions of different types of moon illusions are published, crude estimates might be based upon uncritical polls I have taken just before beginning moon-illusion lectures (see Note 1). Since 1964, over 500 participants have recorded their recollections, first of the comparative distances of the two moons, and then of the "size" comparison. (No hints were given about the distinction between $V$ deg and $S \mathrm{~m}$.) Ballots then were exchanged so that no one reported his or her own responses when the results were tallied by a show of hands.

In a large audience, at least $75 \%$ (and often $90 \%$ ) say the horizon moon usually looked "larger and closer" than the zenith moon. From $5 \%$ to $15 \%$ say "larget and about the same distance." Only about $5 \%$ say "larger and farther." Remaining reports range among the six other possible pair-combinations, even including reports of "smaller and closer" and "smaller and farther."

Reports about memories obviously are poor evidence, but the ones mentioned above do not disagree with published anecdotal "size" and distance reports about the viewed moon. Nor do they disagree with published results for research targets in viewing conditions similar to those for the moon.

6. All circle diameters in Figure 2 are drawn to the same scale, but their distances from point $O^{\prime}$ are drawn at $1 / 10$ th the comparable values; each angle $V^{\prime}$ thus is drawn 10 times larger that its stated value. A uniformly scaled drawing would be obtained by reducing each circle to $1 / 10$ th its present diameter and with the same center. This customary deviation from a uniform scale in moon-illusion diagrams does not affect the present argument.

The choice of a veridical $V^{\prime}$ value for the zenith moon was arbitrary. There are reasons to believe that $V^{\prime}$ is veridical for the horizon moon or, perhaps, for the moon at an elevation of about $20^{\circ}$ (Gilinsky, 1980). Or, to illustrate the "illusion decrement" (see Coren \& Girgus, 1978), prolonged viewing of the moon at any elevation might shift $V^{\prime}$ toward $V$.

7. Baird (1982) clearly differentiated $S^{\prime}$ from $V^{\prime}$, and showed that his "ground model" for explaining changes in $S^{\prime}$ m could not account for the entire moon illusion and that neither could his "sky model" for explaining changes in $V^{\prime}$ deg. Those two models then were merged mathematically into one that describes changes in a single "perceived size" variable: The moon's "perceived size" is determined mostly by ground-model effects when it is near the horizon and mostly by skymodel effects when it is near the zenith, and both effects contribute to the "perceived size" value at middle elevations. The model thus treats $S^{\prime}$ and $V^{\prime}$ as different values of a single variable.

(Manuscript received July 15, 1985; revision accepted for publication December 4,1985 .) 